\title{
An Error Correction Procedure for Multi-Port Measurements at High Frequencies
}

\author{
I. Rolfes and B. Schiek \\ Institut für Hochfrequenztechnik, Ruhr-Universität Bochum, Universitätsstraße 150, 44801 Bochum, Germany
}

\begin{abstract}
The multi-port method for the measurement of the scattering parameters of multi-ports with the help of a vector network analyzer (VNA) with only two measurement ports is presented. For the measurements only two ports of the Nport device can be connected to the two ports of the VNA at a time. The remaining $N-2$ ports of the device under test (DUT) are connected to external terminations. As these external terminations might be mismatched, the measured scattering parameters depend on the reflective characteristics of the terminations. An error correction becomes necessary in order to calculate the effective scattering parameters of the DUT (Tippet and Speciale, 1982; Lu and Chu, 2003).

In contrast to the correction method of Tippet and Speciale (1982), the multi-port method has the advantage, that the external terminations can be chosen arbitrarily. Thus, a realization as an open or short circuit is possible as well. Furthermore, the terminations can be unknown except for one, similar to self-calibration procedures of VNAs. This helps to improve the accuracy of the measurements, because the inconsistency problem due to a slightly erroneous knowledge of the frequency characteristics of the terminations can be eliminated.
\end{abstract}

\section{Introduction}

Although vector network analyzers (VNAs) with more than two measurement ports are already commercially available, VNAs on the basis of two measurement ports and four measurement channels are still widely used in laboratories and industry. For the measurement of a N-port, for example a line coupler or a power divider with three or more ports, with the help of a two-port network analyzer only two ports of the DUT can be connected to the ports of the analyzer at a time. The remaining $N-2$ ports of the DUT are connected to external terminations with the reflection coefficients $\Gamma_{3}, \ldots, \Gamma_{N}$. For the complete determination of the scattering parameters

Correspondence to: I. Rolfes

(Ilona.Rolfes@rub.de) of the device, all possible two-port configurations have to be measured, so that in total

$$
\left(\begin{array}{c}
N \\
2
\end{array}\right)=\frac{N !}{2 !(N-2) !}=\frac{N \cdot(N-1)}{2}
$$

two-port measurements have to be performed. This can be done automatically with the help of switches. In case of perfectly matched terminations, the measured scattering parameters are equal to the effective parameters of the multi-port. For the case of mismatched terminations, the measured scattering parameters depend on the reflective characteristics of the terminations. As a consequence, an error correction becomes necessary. A known method of Tippet and Speciale (1982) shows the following two restrictions. The external terminations have to be known exactly and they cannot be realized as open or short circuits. With the necessity of a precise knowledge of the terminations, inconsistencies due to a slightly erroneous or unprecise knowledge of the reflective characteristics of the terminations as for example of the phase reference planes can lead to considerable errors. This kind of problem is also known from the calibration of network analyzers on the basis of known calibration standards.

\section{The multi-port method}

The scattering parameters of a DUT with $N$ ports, as shown in Fig. 1, are generally described in dependence of the wave parameters as follows:

$$
\left[\begin{array}{c}
b_{1} \\
b_{2} \\
\vdots \\
b_{N}
\end{array}\right]=\mathbf{S}\left[\begin{array}{c}
a_{1} \\
a_{2} \\
\vdots \\
a_{N}
\end{array}\right]
$$

For the measurement of the scattering parameters of such a $N$-port with the help of a VNA with only two ports, it is necessary to perform $h=N \cdot(N-1) / 2$ two-port measurements. Figure 2 shows exemplarily the setup where port 1 and port 2 of the DUT are connected to the VNA while the remaining ports are connected to some external terminations 


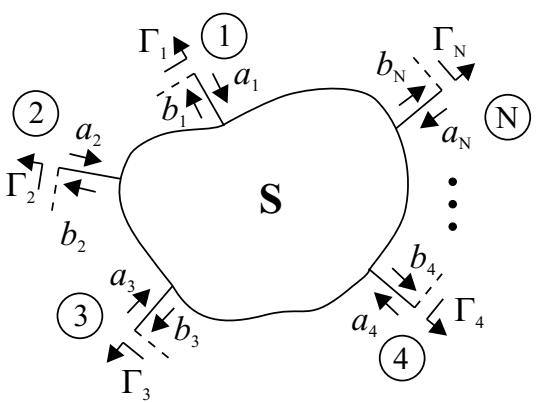

Fig. 1. Wave parameters of a $N$-port.

with the reflection coefficients $\Gamma_{3}, \ldots, \Gamma_{N}$. For this configuration, denoted by the superscript (1) in the following, the wave parameters $a_{i}^{(1)}$ at the ports with the external terminations are represented by: $a_{i}^{(1)}=\Gamma_{i} b_{i}^{(1)}, i=3, \ldots, N$. Next, all further two-port measurements have to be performed as for example with port 1 and port 3 of the DUT being connected to the analyzer.

Table 1 gives an overview of the necessary measurements. The first column specifies a label for the identification of the measurements. In the second column, the ports of the N-port, which are connected to the analyzer, are declared. The third column indicates the terminations at the remaining ports, not being connected to the analyzer. The entirety of these measurements can be represented by the following matrix relation:

$$
\begin{gathered}
{\left[\begin{array}{cccc}
m_{1}^{(1)} & m_{1}^{(2)} & \ldots & b_{1}^{(h)} \\
m_{2}^{(1)} & b_{2}^{(2)} & \ldots & b_{2}^{(h)} \\
b_{3}^{(1)} & m_{3}^{(2)} & \ldots & b_{3}^{(h)} \\
b_{4}^{(1)} & b_{4}^{(2)} & \ldots & b_{4}^{(h)} \\
\vdots & \vdots & \vdots & \vdots \\
b_{N-1}^{(1)} & b_{N-1}^{(2)} & \ldots & m_{N-1}^{(h)} \\
b_{N}^{(1)} & b_{N}^{(2)} & \ldots & m_{N}^{(h)}
\end{array}\right]} \\
=\mathbf{S}\left[\begin{array}{ccccc}
(1) & & & \\
& a_{1}^{(1)} & a_{1}^{(2)} & \ldots & \Gamma_{1} b_{1}^{(h)} \\
a_{2}^{(1)} & & \Gamma_{2} b_{2}^{(2)} & \ldots & \Gamma_{2} b_{2}^{(h)} \\
\Gamma_{3} b_{3}^{(1)} & a_{3}^{(2)} & \ldots & \Gamma_{3} b_{3}^{(h)} \\
\Gamma_{4} b_{4}^{(1)} & \Gamma_{4} b_{4}^{(2)} & \ldots & \Gamma_{4} b_{4}^{(h)} \\
\vdots & & \vdots & \vdots & \vdots \\
\Gamma_{N-1} b_{N-1}^{(1)} & \Gamma_{N-1} b_{N-1}^{(2)} & \ldots & a_{N-1}^{(h)} \\
\Gamma_{N} b_{N}^{(1)} & \Gamma_{N} b_{N}^{(2)} & \ldots & a_{N}^{(h)}
\end{array}\right]
\end{gathered}
$$

The wave parameters, known from the measurements with the calibrated analyzer, are denoted by $m_{i}$ and $a_{i}$. The unknown parameters, the wave parameters $b_{i}$ and the matrix $\mathbf{S}$, will be determined with the help of the multi-port method. For this purpose, the reflection coefficients $\Gamma_{1}, \ldots, \Gamma_{N}$ are assumed to be known initially.

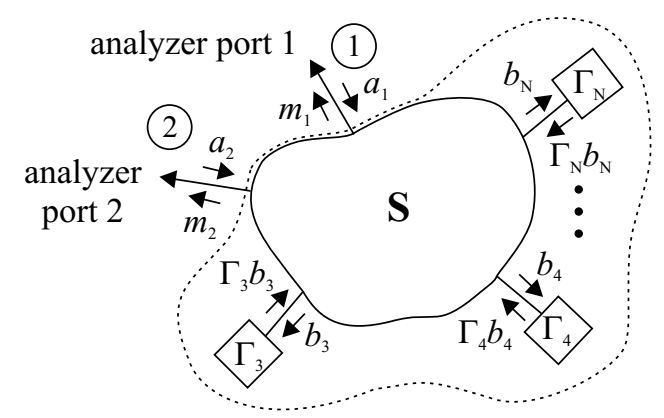

Fig. 2. Setup for the measurement of the scattering parameters of a $N$-port with a 2-port-VNA.

\subsection{The multi-port method for a three-port}

The theory for a DUT with 3 ports will at first be derived. Equation (3) can be written as:

$$
\begin{aligned}
& \underbrace{\left[\begin{array}{ccc}
b_{1}^{(1)} & 0 & 0 \\
0 & b_{2}^{(2)} & 0 \\
0 & 0 & b_{3}^{(3)}
\end{array}\right]}_{\mathbf{b}}+\underbrace{\left[\begin{array}{ccc}
0 & m_{1}^{(2)} & m_{1}^{(3)} \\
m_{2}^{(1)} & 0 & m_{2}^{(3)} \\
m_{3}^{(1)} & m_{3}^{(2)} & 0
\end{array}\right]}_{\mathbf{M}_{\mathbf{1}}} \\
& =\mathbf{S} \underbrace{\left[\begin{array}{ccc}
\Gamma_{1} b_{1}^{(1)} & 0 & 0 \\
0 & \Gamma_{2} b_{2}^{(2)} & 0 \\
0 & 0 & \Gamma_{3} b_{3}^{(3)}
\end{array}\right]}_{\Gamma \mathbf{b}}+\underbrace{\left[\begin{array}{ccc}
0 & a_{1}^{(2)} & a_{1}^{(3)} \\
a_{2}^{(1)} & 0 & a_{2}^{(3)} \\
a_{3}^{(1)} & a_{3}^{(2)} & 0
\end{array}\right]}_{\mathbf{M}_{\mathbf{2}}},
\end{aligned}
$$

resulting in

$\mathbf{b}+\mathbf{M}_{\mathbf{1}}=\mathbf{S}\left(\Gamma \mathbf{b}+\mathbf{M}_{\mathbf{2}}\right)$

i.e. a partitioning into known values, as the measurement matrices $\mathbf{M}_{1}, \mathbf{M}_{2}$ and the reflection matrix $\Gamma$, and unknown values, namely the scattering matrix $\mathbf{S}$ and the wave parameter matrix $\mathbf{b}$. With the additional microwave excitations $\tilde{a}_{1}^{(2)}, \tilde{a}_{1}^{(3)}, \tilde{a}_{2}^{(1)}, \tilde{a}_{2}^{(3)}, \tilde{a}_{3}^{(1)}, \tilde{a}_{3}^{(2)}$, which are different from the previous excitations in Eq. (4), a further equation, similar to Eq. (5) can be derived:

$\tilde{\mathbf{b}}+\tilde{\mathbf{M}}_{\mathbf{1}}=\mathbf{S}\left(\Gamma \tilde{\mathbf{b}}+\tilde{\mathbf{M}}_{\mathbf{2}}\right)$.

In order to determine the unknown parameters, the scattering matrix $\mathbf{S}$ is eliminated, so that the unknown wave parameters can be calculated at first. A manipulation of the matrix equations leads to:

$\left(\Gamma \mathbf{b}+\mathbf{M}_{2}\right)\left(\mathbf{M}_{2}-\Gamma \mathbf{M}_{1}\right)^{-1}=(\mathbf{I}-\Gamma \mathbf{S})^{-1}$
$\left(\Gamma \tilde{\mathbf{b}}+\tilde{\mathbf{M}}_{\mathbf{2}}\right)\left(\tilde{\mathbf{M}}_{\mathbf{2}}-\Gamma \tilde{\mathbf{M}}_{\mathbf{1}}\right)^{-1}=(\mathbf{I}-\Gamma \mathbf{S})^{-1}$

resulting in

$$
\begin{aligned}
& \left(\Gamma \mathbf{b}+\mathbf{M}_{2}\right)\left(\mathbf{M}_{2}-\Gamma \mathbf{M}_{1}\right)^{-1} \\
& =\left(\Gamma \tilde{\mathbf{b}}+\tilde{\mathbf{M}}_{\mathbf{2}}\right)\left(\tilde{\mathbf{M}}_{\mathbf{2}}-\Gamma \tilde{\mathbf{M}}_{1}\right)^{-1} .
\end{aligned}
$$

On the basis of this equation it could be supposed that the parameter $\mathbf{b}$ cannot be determined for the case $\Gamma_{i}=0, i=1,2,3$. 
Table 1. Overview of the multi-port measurements.

\begin{tabular}{ccc}
\hline measurement & port at the VNA & terminations \\
\hline$(1)$ & 1,2 & $\Gamma_{3}, \ldots, \Gamma_{N}$ \\
$(2)$ & 1,3 & $\Gamma_{2}, \Gamma_{4}, \ldots, \Gamma_{N}$ \\
$\vdots$ & $\vdots$ & $\vdots$ \\
$(N-1)$ & $1, N$ & $\Gamma_{2}, \ldots, \Gamma_{N-1}$ \\
$(N)$ & 2,3 & $\Gamma_{1}, \Gamma_{4}, \ldots, \Gamma_{N}$ \\
$\vdots$ & $\vdots$ & $\vdots$ \\
$(2 N-3)$ & $2, N$ & $\Gamma_{1}, \Gamma_{3}, \ldots, \Gamma_{N-1}$ \\
$\vdots$ & $\vdots$ & $\vdots$ \\
$(h)=(N \cdot(N-1) / 2)$ & $N-1, N$ & $\Gamma_{1}, \ldots, \Gamma_{N-2}$
\end{tabular}

But it is possible to eliminate the singularity for $\Gamma_{i}=0$, so that the following solutions result after some manipulations:

$$
\begin{aligned}
b_{1}^{(1)}= & \left(\Gamma _ { 3 } ^ { ( 1 ) } \left(\Gamma_{2}^{(3)}\left(a_{1}^{(2)} \tilde{m}_{1}^{(3)}-\tilde{a}_{1}^{(3)} m_{1}^{(2)}\right)+\tilde{\Gamma}_{2}^{(3)}\left(a_{1}^{(3)} m_{1}^{(2)}\right.\right.\right. \\
& \left.\left.\left.-a_{1}^{(2)} m_{1}^{(3)}\right)\right)+\Gamma_{2}^{(1)} \Gamma_{3}^{(2)}\left(a_{1}^{(3)} \tilde{m}_{1}^{(3)}-\tilde{a}_{1}^{(3)} m_{1}^{(3)}\right)\right) / D_{1} \\
b_{2}^{(2)}= & \left(\Gamma _ { 1 } ^ { ( 2 ) } \left(\Gamma_{3}^{(1)}\left(a_{2}^{(3)} \tilde{m}_{2}^{(1)}-\tilde{a}_{2}^{(1)} m_{2}^{(3)}\right)+\tilde{\Gamma}_{3}^{(1)}\left(a_{2}^{(1)} m_{2}^{(3)}\right.\right.\right. \\
& \left.\left.\left.-a_{2}^{(3)} m_{2}^{(1)}\right)\right)+\Gamma_{1}^{(3)} \Gamma_{3}^{(2)}\left(a_{2}^{(1)} \tilde{m}_{2}^{(1)}-\tilde{a}_{2}^{(1)} m_{2}^{(1)}\right)\right) / D_{2} \\
b_{3}^{(3)}= & \left(\Gamma _ { 2 } ^ { ( 3 ) } \left(\Gamma_{1}^{(2)}\left(\tilde{a}_{3}^{(2)} m_{3}^{(1)}-a_{3}^{(1)} \tilde{m}_{3}^{(2)}\right)+\tilde{\Gamma}_{1}^{(2)}\left(a_{3}^{(1)} m_{3}^{(2)}\right.\right.\right. \\
& \left.\left.\left.-a_{3}^{(2)} m_{3}^{(1)}\right)\right)+\Gamma_{2}^{(1)} \Gamma_{1}^{(3)}\left(\tilde{a}_{3}^{(2)} m_{3}^{(2)}-a_{3}^{(2)} \tilde{m}_{3}^{(2)}\right)\right) / D_{3}
\end{aligned}
$$

with $i=1,2,3, j=1,2,3, i \neq j$

$\Gamma_{i}^{(j)}=a_{i}^{(j)}-\Gamma_{i} m_{i}^{(j)}, \quad \tilde{\Gamma}_{i}^{(j)}=\tilde{a}_{i}^{(j)}-\Gamma_{i} \tilde{m}_{i}^{(j)}$

and the denominators

$$
\begin{aligned}
& D_{1}=\left(\Gamma_{3}^{(2)}\left(\Gamma_{1}^{(3)} \tilde{\Gamma}_{2}^{(3)}-\tilde{\Gamma}_{1}^{(3)} \Gamma_{2}^{(3)}\right)\right) \\
& D_{2}=\left(\Gamma_{1}^{(3)}\left(\Gamma_{2}^{(1)} \tilde{\Gamma}_{3}^{(1)}-\tilde{\Gamma}_{2}^{(1)} \Gamma_{3}^{(1)}\right)\right) \\
& D_{3}=\left(\Gamma_{2}^{(1)}\left(\Gamma_{1}^{(2)} \tilde{\Gamma}_{3}^{(2)}-\tilde{\Gamma}_{1}^{(2)} \Gamma_{3}^{(2)}\right)\right)
\end{aligned}
$$

On the basis of these equations the matrix $\mathbf{S}$ can be determined with the help of Eqs. (3) or (4) respectively. In comparison to the method of Tippet and Speciale (1982) the terminations $\Gamma_{1}, \ldots, \Gamma_{N}$ can be realized arbitrarily, i.e. $\Gamma_{i}= \pm 1$ is also possible. Further on, the terminations can be unknown except for one.

\subsection{Multi-port method with unknown terminations}

The reflection coefficients of the external terminations are calculable on the basis of the following equations:

$\Gamma_{2}=\frac{h_{11}+\Gamma_{1} h_{12}}{h_{13}+\Gamma_{1} h_{14}}, \quad \Gamma_{3}=\frac{h_{21}+\Gamma_{1} h_{22}}{h_{23}+\Gamma_{1} h_{24}}$, where $\Gamma_{1}$ has to be known and the parameters $h_{i j}$ are known from measurements with:

$$
\begin{array}{ll}
h_{11}=\tilde{a}_{1}^{(2)} \tilde{h}_{15}-a_{1}^{(2)} h_{15}, & h_{12}=m_{1}^{(2)} h_{15}-\tilde{m}_{1}^{(2)} \tilde{h}_{15}, \\
h_{13}=a_{1}^{(2)} h_{16}-\tilde{a}_{1}^{(2)} \tilde{h}_{16}, & h_{14}=\tilde{m}_{1}^{(2)} \tilde{h}_{16}-m_{1}^{(2)} h_{16}, \\
h_{15}=a_{2}^{(1)} \tilde{h}_{18}-\tilde{a}_{2}^{(1)} \tilde{h}_{17}, \quad \tilde{h}_{15}=a_{2}^{(1)} h_{17}-\tilde{a}_{2}^{(1)} h_{18}, \\
h_{16}=\tilde{m}_{2}^{(1)} \tilde{h}_{17}-m_{2}^{(1)} \tilde{h}_{18}, \quad \tilde{h}_{16}=\tilde{m}_{2}^{(1)} h_{18}-m_{2}^{(1)} h_{17}, \\
h_{17}=a_{3}^{(2)} \tilde{m}_{3}^{(1)}-\tilde{a}_{3}^{(1)} m_{3}^{(2)}, \tilde{h}_{17}=\tilde{a}_{3}^{(2)} m_{3}^{(1)}-a_{3}^{(1)} \tilde{m}_{3}^{(2)}, \\
h_{18}=a_{3}^{(2)} m_{3}^{(1)}-a_{3}^{(1)} m_{3}^{(2)}, \tilde{h}_{18}=\tilde{a}_{3}^{(2)} \tilde{m}_{3}^{(1)}-\tilde{a}_{3}^{(1)} \tilde{m}_{3}^{(2)}
\end{array}
$$

and

$$
\begin{array}{ll}
h_{21}=\tilde{a}_{3}^{(1)} \tilde{h}_{25}-a_{3}^{(1)} h_{25}, & h_{22}=\tilde{a}_{3}^{(1)} \tilde{h}_{26}-a_{3}^{(1)} h_{26}, \\
h_{23}=\tilde{m}_{3}^{(1)} \tilde{h}_{25}-m_{3}^{(1)} h_{25}, & h_{24}=\tilde{m}_{3}^{(1)} \tilde{h}_{26}-m_{3}^{(1)} h_{26}, \\
h_{25}=a_{1}^{(3)} \tilde{h}_{28}-\tilde{a}_{1}^{(3)} h_{27}, & \tilde{h}_{25}=a_{1}^{(3)} \tilde{h}_{27}-\tilde{a}_{1}^{(3)} h_{28}, \\
h_{26}=\tilde{m}_{1}^{(3)} h_{27}-m_{1}^{(3)} \tilde{h}_{28}, & \tilde{h}_{26}=\tilde{m}_{1}^{(3)} h_{28}-m_{1}^{(3)} \tilde{h}_{27}, \\
h_{27}=\tilde{a}_{2}^{(1)} m_{2}^{(3)}-a_{2}^{(3)} \tilde{m}_{2}^{(1)}, \tilde{h}_{27}=a_{2}^{(1)} \tilde{m}_{2}^{(3)}-\tilde{a}_{2}^{(3)} m_{2}^{(1)}, \\
h_{28}=a_{2}^{(1)} m_{2}^{(3)}-a_{2}^{(3)} m_{2}^{(1)}, \tilde{h}_{28}=\tilde{a}_{2}^{(1)} \tilde{m}_{2}^{(3)}-\tilde{a}_{2}^{(3)} \tilde{m}_{2}^{(1)} .
\end{array}
$$

This is similar to self-calibration procedures for VNAs, where the calibration standards can be partly unknown. The frequency characteristics of the terminations are determined within the multi-port procedure. The previously described problem of inconsistencies is reduced dramatically.

\subsection{Multi-port method for N-ports}

These results can be applied to a device with multiple ports by subdividing the device into

$$
\left(\begin{array}{c}
N \\
3
\end{array}\right)=\frac{N !}{3 !(N-3) !}=\frac{N \cdot(N-1) \cdot(N-2)}{6}
$$

sub-devices with three connectors as shown in Fig. 3. The ports 4 to $N$ of the $\mathrm{N}$-port are connected to the terminations $\Gamma_{4}, \ldots, \Gamma_{N}$ and the resulting network with the ports 1,2 and 3 can be considered as a 3 -port with the scattering matrix $\mathbf{S}_{\mathbf{1}}$ as shown by the dashed line in Fig. 3. For this 3-port device, the 2-port measurements at ports 1 and 2, 1 and 3 as well as 2 and 3 are used for the calculations. These measurements can also be identified as the measurements (1), (2) and $(N)$ according to Table 1 . The following matrix representation of the 3-port device in dependence of the scattering matrix $\mathbf{S}_{\mathbf{1}}$ results according to Eq. (4):

$$
\left[\begin{array}{ccc}
b_{1}^{(N)} & m_{1}^{(2)} & m_{1}^{(1)} \\
m_{2}^{(N)} & b_{2}^{(2)} & m_{2}^{(1)} \\
m_{3}^{(N)} & m_{3}^{(2)} & b_{3}^{(1)}
\end{array}\right]=\mathbf{S}_{\mathbf{1}}\left[\begin{array}{ccc}
\Gamma_{1} b_{1}^{(N)} & a_{1}^{(2)} & a_{1}^{(1)} \\
a_{2}^{(N)} & \Gamma_{2} b_{2}^{(2)} & a_{2}^{(1)} \\
a_{3}^{(N)} & a_{3}^{(2)} & \Gamma_{3} b_{3}^{(1)}
\end{array}\right]
$$

By the application of the 3-port method with Eqs. (10)-(12) the unknown wave parameters $b_{1}^{(N)}, b_{2}^{(2)}$ and $b_{3}^{(1)}$ are calculable. The previously derived theory with partly unknown reflective terminations can as well be adopted. For the determination of the complete unknown scattering matrix $\mathbf{S}$ of the $\mathrm{N}$-port device it is necessary to determine the whole set of wave parameters $b$ as defined in Fig. 3. These parameters can be derived similarly by considering the remaining 3 -port combinations of the $\mathrm{N}$-port as well as the associated equations. 


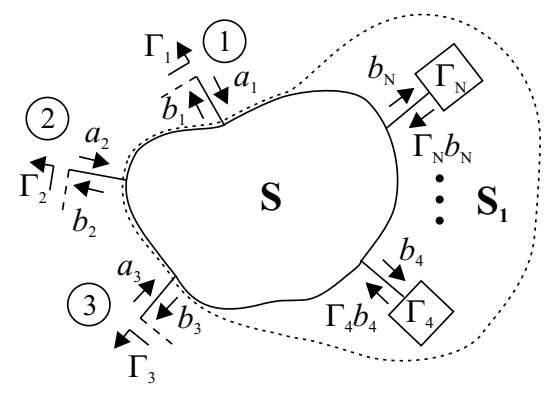

Fig. 3. Subdivision of a $N$-port into a 3-port.

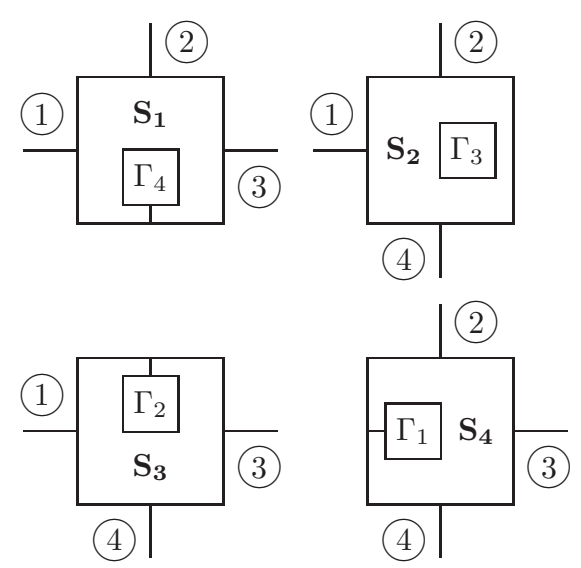

Fig. 4. Possible three-port configurations of a four-port device.

In the following this approach will be discussed for the example of a 4-port with the unknown scattering matrix $\mathbf{S}$. From the measurement of the scattering parameters of the 4-port with a 2-port VNA the following six relations result:

$$
\begin{aligned}
& {\left[\begin{array}{c}
m_{1}^{(1)} \\
m_{2}^{(1)} \\
b_{3}^{(1)} \\
b_{4}^{(1)}
\end{array}\right]=\mathbf{S}\left[\begin{array}{c}
a_{1}^{(1)} \\
a_{2}^{(1)} \\
\Gamma_{3} b_{3}^{(1)} \\
\Gamma_{4} b_{4}^{(1)}
\end{array}\right],\left[\begin{array}{c}
m_{1}^{(2)} \\
b_{2}^{(2)} \\
m_{3}^{(2)} \\
b_{4}^{(2)}
\end{array}\right]=\mathbf{S}\left[\begin{array}{c}
a_{1}^{(2)} \\
\Gamma_{2} b_{2}^{(2)} \\
a_{3}^{(2)} \\
\Gamma_{4} b_{4}^{(2)}
\end{array}\right],} \\
& {\left[\begin{array}{c}
m_{1}^{(3)} \\
b_{2}^{(3)} \\
b_{3}^{(3)} \\
m_{4}^{(3)}
\end{array}\right]=\mathbf{S}\left[\begin{array}{c}
a_{1}^{(3)} \\
\Gamma_{2} b_{2}^{(3)} \\
\Gamma_{3} a_{3}^{(3)} \\
a_{4}^{(3)}
\end{array}\right],\left[\begin{array}{c}
b_{1}^{(4)} \\
m_{2}^{(4)} \\
m_{3}^{(4)} \\
b_{4}^{(4)}
\end{array}\right]=\mathbf{S}\left[\begin{array}{c}
\Gamma_{1} b_{1}^{(4)} \\
a_{2}^{(4)} \\
a_{3}^{(4)} \\
\Gamma_{4} b_{4}^{(4)}
\end{array}\right],} \\
& {\left[\begin{array}{c}
b_{1}^{(5)} \\
m_{2}^{(5)} \\
b_{3}^{(5)} \\
m_{4}^{(5)}
\end{array}\right]=\mathbf{S}\left[\begin{array}{c}
\Gamma_{1} b_{1}^{(5)} \\
a_{2}^{(5)} \\
\Gamma_{3} b_{3}^{(5)} \\
a_{4}^{(5)}
\end{array}\right],\left[\begin{array}{c}
b_{1}^{(6)} \\
b_{2}^{(6)} \\
m_{3}^{(6)} \\
m_{4}^{(6)}
\end{array}\right]=\mathbf{S}\left[\begin{array}{c}
\Gamma_{1} b_{1}^{(6)} \\
\Gamma_{2} b_{2}^{(6)} \\
a_{3}^{(6)} \\
a_{4}^{(6)}
\end{array}\right],}
\end{aligned}
$$

leading to the following matrix representation:

$$
\left[\begin{array}{cccccc}
m_{1}^{(1)} & m_{1}^{(2)} & m_{1}^{(3)} & b_{1}^{(4)} & b_{1}^{(5)} & b_{1}^{(6)} \\
m_{2}^{(1)} & b_{2}^{(2)} & b_{2}^{(3)} & m_{2}^{(4)} & m_{2}^{(5)} & b_{2}^{(6)} \\
b_{3}^{(1)} & m_{3}^{(2)} & b_{3}^{(3)} & m_{3}^{(4)} & b_{3}^{(5)} & m_{3}^{(6)} \\
b_{4}^{(1)} & b_{4}^{(2)} & m_{4}^{(3)} & b_{4}^{(4)} & m_{4}^{(5)} & m_{4}^{(6)}
\end{array}\right]
$$

$$
=\mathbf{S}\left[\begin{array}{cccccc}
a_{1}^{(1)} & a_{1}^{(2)} & a_{1}^{(3)} & \Gamma_{1} b_{1}^{(4)} & \Gamma_{1} b_{1}^{(5)} & \Gamma_{1} b_{1}^{(6)} \\
a_{2}^{(1)} & \Gamma_{2} b_{2}^{(2)} & \Gamma_{2} b_{2}^{(3)} & a_{2}^{(4)} & a_{2}^{(5)} & \Gamma_{2} b_{2}^{(6)} \\
\Gamma_{3} b_{3}^{(1)} & a_{3}^{(2)} & \Gamma_{3} b_{3}^{(3)} & a_{3}^{(4)} & \Gamma_{3} b_{3}^{(5)} & a_{3}^{(6)} \\
\Gamma_{4} b_{4}^{(1)} & \Gamma_{4} b_{4}^{(2)} & a_{4}^{(3)} & \Gamma_{4} b_{4}^{(4)} & a_{4}^{(5)} & a_{4}^{(6)}
\end{array}\right]
$$

This relation represents a linear system of equations for the determination of the unknown scattering matrix $\mathbf{S}$ of the 4port. For the calculation of the unknown parameters $b$ in Eq. (21) the 3-port theory is applied. For example, the 4port is terminated at its port 4 by the reflection $\Gamma_{4}$ as shown in Fig. 4. The resulting 3-port with the terminals 1, 2, 3 is described by the scattering matrix $\mathbf{S}_{\mathbf{1}}$ with:

$$
\left[\begin{array}{ccc}
b_{1}^{(4)} & m_{1}^{(2)} & m_{1}^{(1)} \\
m_{2}^{(4)} & b_{2}^{(2)} & m_{2}^{(1)} \\
m_{3}^{(4)} & m_{3}^{(2)} & b_{3}^{(1)}
\end{array}\right]=\mathbf{S}_{\mathbf{1}}\left[\begin{array}{ccc}
\Gamma_{1} b_{1}^{(4)} & a_{1}^{(2)} & a_{1}^{(1)} \\
a_{2}^{(4)} & \Gamma_{2} b_{2}^{(2)} & a_{2}^{(1)} \\
a_{3}^{(4)} & a_{3}^{(2)} & \Gamma_{3} b_{3}^{(1)}
\end{array}\right] .
$$

On the basis of the 3-port algorithm the wave parameters $b_{1}^{(4)}, b_{2}^{(2)}$ and $b_{3}^{(1)}$ can be determined. Accordingly, the 4port can be subdivided into three further 3-ports as shown in Fig. 4. The scattering matrices are denoted by $\mathbf{S}_{\mathbf{2}}, \mathbf{S}_{\mathbf{3}}, \mathbf{S}_{\mathbf{4}}$.

$$
\begin{aligned}
& {\left[\begin{array}{lll}
b_{1}^{(5)} & m_{1}^{(3)} & m_{1}^{(1)} \\
m_{2}^{(5)} & b_{2}^{(3)} & m_{2}^{(1)} \\
m_{4}^{(5)} & m_{4}^{(3)} & b_{4}^{(1)}
\end{array}\right]=\mathbf{S}_{\mathbf{2}}\left[\begin{array}{ccc}
\Gamma_{1} b_{1}^{(5)} & a_{1}^{(3)} & a_{1}^{(1)} \\
a_{2}^{(5)} & \Gamma_{2} b_{2}^{(3)} & a_{2}^{(1)} \\
a_{4}^{(5)} & a_{4}^{(3)} & \Gamma_{4} b_{4}^{(1)}
\end{array}\right]} \\
& {\left[\begin{array}{ccc}
b_{1}^{(6)} & m_{1}^{(3)} & m_{1}^{(2)} \\
m_{3}^{(6)} & b_{3}^{(3)} & m_{3}^{(2)} \\
m_{4}^{(6)} & m_{4}^{(3)} & b_{4}^{(2)}
\end{array}\right]=\mathbf{S}_{\mathbf{3}}\left[\begin{array}{ccc}
\Gamma_{1} b_{1}^{(6)} & a_{1}^{(3)} & a_{1}^{(2)} \\
a_{3}^{(6)} & \Gamma_{3} b_{3}^{(3)} & a_{3}^{(2)} \\
a_{4}^{(6)} & a_{4}^{(3)} & \Gamma_{4} b_{4}^{(2)}
\end{array}\right]} \\
& {\left[\begin{array}{ccc}
b_{2}^{(6)} & m_{2}^{(5)} & m_{2}^{(4)} \\
m_{3}^{(6)} & b_{3}^{(5)} & m_{3}^{(4)} \\
m_{4}^{(6)} & m_{4}^{(5)} & b_{4}^{(4)}
\end{array}\right]=\mathbf{S}_{\mathbf{4}}\left[\begin{array}{ccc}
\Gamma_{2} b_{2}^{(6)} & a_{2}^{(5)} & a_{2}^{(4)} \\
a_{3}^{(6)} & \Gamma_{3} b_{3}^{(5)} & a_{3}^{(4)} \\
a_{4}^{(6)} & a_{4}^{(5)} & \Gamma_{4} b_{4}^{(4)}
\end{array}\right]}
\end{aligned}
$$

The unknown wave parameters can thus be calculated, so that finally on the basis of Eq. (21) the scattering matrix $\mathbf{S}$ of the 4-port can be determined completely. The redundancy in the number of equations can be used for a least-square-error minimization in order to enhance the precision of the method. As explained before, three of the external terminations can be unknown and will be determined during the multi-port procedure.

\subsection{Extension of the multi-port method}

With the multi-port method the scattering parameters of a $\mathrm{N}$-port are determined on the basis of a VNA with two measurement ports and furthermore $N$ terminations which can be unknown except for one. In order to avoid the demand for one known external termination, which is measurable with a calibrated VNA, it is also possible to determine the characteristics of this termination in combination with the measurements of the DUT by performing one additional reflection measurement. For this purpose, one further 1-port measurement has to be performed in addition to the previously mentioned two-port measurements of the DUT. For example considering the setup for the two-port measurements in Fig. 2, port 1 and port 2 of the DUT are connected to the 

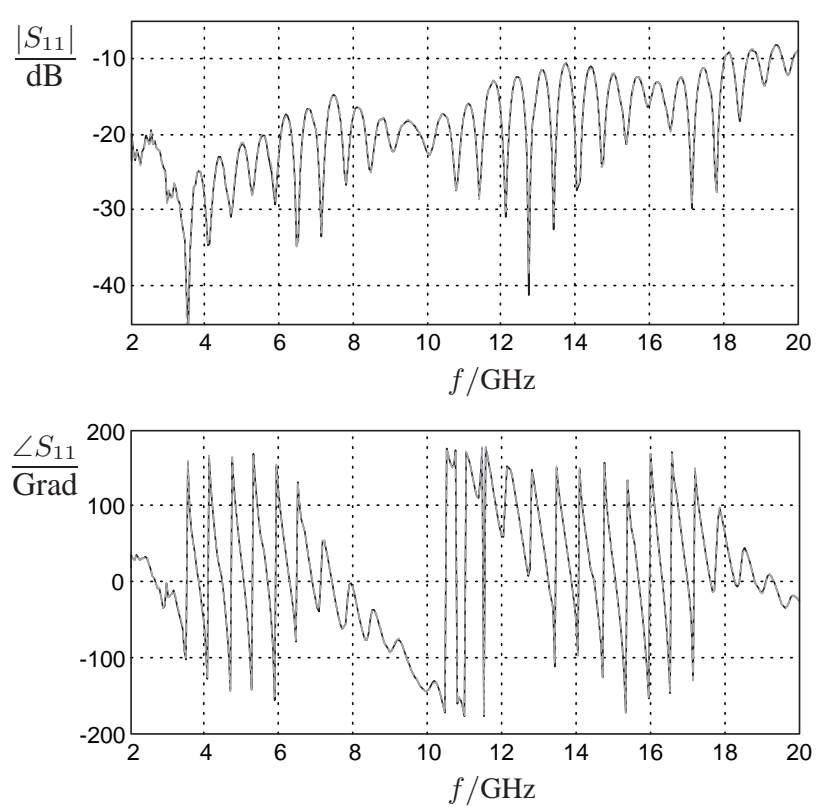

Fig. 5. Error-corrected scattering parameter $S_{11}$ with reflective (- -) and matched (-) terminations.

analyzer and the remaining ports are terminated with the reflections $\Gamma_{3}, \ldots, \Gamma_{N}$. For this configuration the scattering parameters of the two-port between the connectors 1 and 2, denoted by $S_{m 11}, S_{m 12}, S_{m 21}, S_{m 22}$, are measured. Terminating additionally port 1 of the DUT with the reflection coefficient $\Gamma_{1}$ and performing a 1-port measurement at port 2 of the DUT leads to a measurement result for $\Gamma_{m 2}$ with

$\Gamma_{m 2}=S_{m 22}+\frac{S_{m 21} S_{m 12} \Gamma_{1}}{1-S_{m 11} \Gamma_{1}}$.

This relation can be solved for $\Gamma_{1}$, so that the reflection coefficient at port 1 can be determined from one additional 1-port measurement in combination with the connection of the DUT. However, determining all reflection coefficients of the external terminations from such reflection measurements instead of calculating them from Eq. (17), numerous additional measurements would have to be performed, enlarging the measurement complexity considerably.

\section{Experimental Results}

Several measurements with various multi-port devices were performed with the help of a 2-port VNA of the type HP8510C. In the following the measured results for a commercial multistage line coupler with coaxial connectors are presented exemplarily. The calibration of the VNA was performed on the basis of a TRL-calibration. In Figs. 5 to 7 the measured scattering parameters $S_{11}, S_{12}$ and $S_{14}$ of the DUT are depicted according to their absolute values and their phases in a frequency range from $2 \mathrm{GHz}$ to $20 \mathrm{GHz}$. The error correction of the measured scattering parameters was
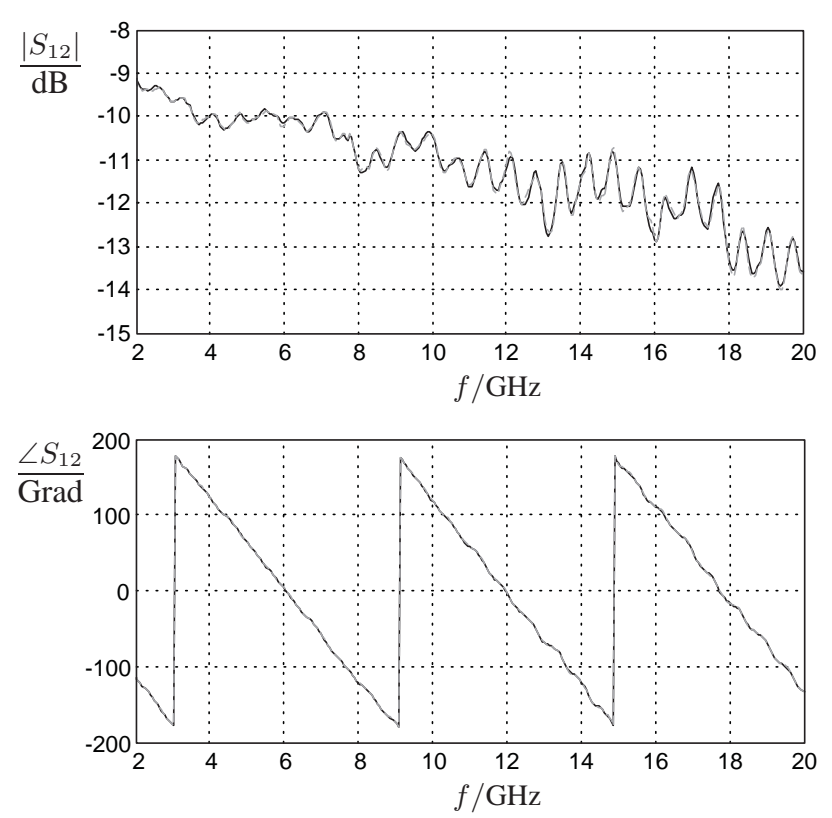

Fig. 6. Error-corrected scattering parameter $S_{12}$ with reflective (- -) and matched $(-)$ terminations.
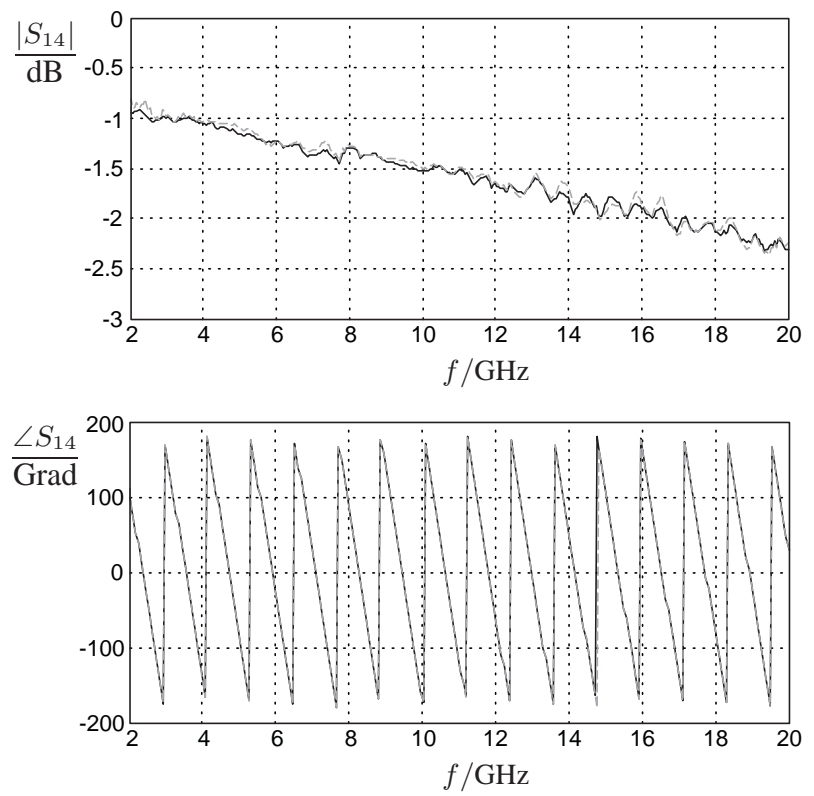

Fig. 7. Error-corrected scattering parameter $S_{14}$ with reflective (- -) and matched (-) terminations.

performed according to the multi-port method with mostly unknown reflective terminations in comparison to the measurement with matched terminations. The measured results show a good agreement between both methods. 


\section{Conclusion}

The theory of the multi-port method for the measurement of the scattering parameters of a $\mathrm{N}$-port device with the help of a VNA with only two measurement ports and its experimental application have been presented. In contrast to the known correction method for the measurement of multi-port devices of Tippet and Speciale (1982), the developed multiport method has the advantage that it is based on the measurement with external terminations which can be chosen arbitrarily as long as the topology of the DUT allows it. In addition, these external terminations can be unknown except for one, enhancing the accuracy of the error correction procedure. The inconsistency problem due to a slightly erroneous knowledge about the frequency characteristics of the terminations can be eliminated, similarly to self-calibration procedures for VNAs.

\section{References}

Lu, H.-C. and Chu, T.-H.: Multiport Scattering Matrix Measurement Using a Reduced-Port Network Analyzer, IEEE Trans. Microw. Theory Tech., vol. MTT-51, 1525-1533, May, 2003.

Tippet, J. C. and Speciale, R. A.: A Rigorous Technique for Measuring the Scattering Matrix of a Multiport Device with a 2Port Network Analyzer, IEEE Trans. Microw. Theory Tech., vol. MTT-30, 661-666, May, 1982. 